\title{
Routing Protocols under different Mobility Models, Node Density and Speed
}

\author{
${ }^{1}$ Deepak Kumar and Suresh C. Gupta \\ Department of Electrical Engineering, Indian Institute of Technology (BHU), Varanasi, India \\ ${ }^{1}\{$ dkumar.rs.eee, scgupta.eee\}@iitbhu.ac.in
}

\begin{abstract}
Mobile Ad hoc NETwork (MANET) is self-organized and decentralized network. Due to these properties, they are usable in the field of disaster management, war scenarios, vehicular network etc. To send packets from source to destination, route(s) are required; to achieve this task routing protocols are designed. Routing protocols for MANET have been grouped into four types on the basis of single channel classification. These are Proactive, Reactive, Hybrid and Location-based/ Geographic. The performance of these protocols depends on various factors including mobility pattern/model, speed of node, density of node, area, transmission range etc. In this paper the factors taken into account are mobility models, speed of node and density of node. The performance metrics includes throughput, end to end delay and packet loss. The protocols simulated are DSR, LAR, OLSR and ZRP, choosing one from each group discussed above. The mobility models employed in this study are Random Way Point, Gauss Markov, Reference Point Group Mobility and Manhattan
\end{abstract} Grid.

From the results it is evident that with the change in mobility pattern, speed and density of the nodes the performance varies. Furthermore it has been observed that location based routing protocols (LAR) and OLSR shows good performance with these variations.

Keywords: MANET, DSR, LAR, OLSR, ZRP, Random Waypoint mobility model, Gauss Markov model, Manhattan Grid model, RPGM, End to end delay, Throughput, Packet loss

\section{Introduction}

Mobile Ad-hoc NETwork (MANET) is an infrastructure less network which can be rapidly deployed with improved flexibility and reduced cost. A node in the network receives data from source node in a multi-hop pattern. The nodes are free to move randomly and may join or leave the network. Due to this element of randomness, the network topology becomes unpredictable and may change rapidly. A routing protocol is implied to find a path from source to destination and many have been proposed in literature so far. These protocols are further divided into four groups depending on their method to find a route.

1. Proactive protocol: The route information is obtained a priori and stored in a table for future lookup. E.g. OLSR [3].

2. Reactive protocol: The route information is calculated when and wherever required hence favoring an on demand route formation. E.g. DSR [1].

3. Hybrid protocol: It combines the advantages of proactive and reactive routing. The route is initially established with some proactive protocol and then to serve the 
demands from additionally activated nodes are responded with reactive flooding. E.g. ZRP [4].

4. Geographical Protocol: The route information is obtained using the location information of nodes. E.g. LAR [2].

There are many challenges with MANET. Due to node mobility links can be made or breached owing to limit in range of communication. The routing protocol should quickly adapt to network changes and find new paths that avoid the failed links. But this is a difficult task due to constraints like low wireless bandwidth and limited battery power of nodes. This overhead can have a significant impact on the overall performance of the network.

The movement of the nodes can be possibly defined by a mobility model. A lot of models have been proposed so far including Random waypoint model [5], Gauss Markov [6], Manhattan Grid [8], Reference point group mobility[7] etc. Each of these models has different ways to define the possible movements of node. The choice of mobility model affects the performance of routing protocols.

These networks are suitable in under water networks and emergency situations like disaster management, war scenarios etc.

A variety of comparative studies have been carried out on routing protocols and mobility models using metrics like packet delivery ratio, end to end delay, packet loss and energy consumption. But there are other factors also, on which the performance depends including traffic pattern, network topology and obstacle positions etc. For the sake of simplicity many studies have used Random way point model, to evaluate the performance of protocols.

In this study, we present a comparative study of Proactive, Reactive, hybrid and geographic protocols. The comparative studies available mostly use random waypoint which is an unrealistic approach due to its probabilistic nature. We have considered three other mobility models along with random waypoint model for a realistic behavior. We have considered variation in node density and node velocity. Studies are carried out in GloMoSim simulator which provides a scalable simulation environment for wireless networks.

\section{Routing Protocols}

The Ad hoc routing protocols, which we tested in our simulation experiments covers all the four category viz. Proactive, Reactive, Hybrid and Geographic routing. The functionality of each protocol is discussed below.

\subsection{DSR (Dynamic Source Routing) [1]}

It is a reactive protocol. It is designed specifically for use in multi-hop wireless networks. It comprises two mechanisms namely Route maintenance and Route discovery. These two processes work together, to allow nodes to discover and maintain routes to destinations.

Route Discovery: It is the mechanism by which a source node $S$ which wants to send a data packet to destination $D$, requests for and obtains a route to $D$. It happens only when route to $D$ is unknown to S.Route Maintenance: It is the mechanism by which source node $S$ is able to detect if there is a topology change, which results in route breakage to destination. When unavailability of a route is shown, the source $S$ can either attempt to use any other route to $D$ which is known by $S$ or can revoke route discovery again to find a new route. 


\subsection{LAR (Location Aided Routing) [2]}

It is a geographic / location based routing protocol. It was built on the notion of reducing the control message overhead of Ad hoc on demand distance vector (AODV) routing protocol and Dynamic Source Routing protocol (DSR). To achieve this, source node $S$ floods packet selectively to only a portion of the network (called Request Zone) which is likely to have the route to destination D. To identify such location, it utilizes Global Positioning System (GPS). LAR assumes that:

1. Every node in the network knows the speed of corresponding destination node.

2. Every node in the network knows the location of corresponding destination node.

It was proposed by Y.B. Ko et.al [6]. Two concepts were proposed, namely LAR Box (LAR 1) and LAR Step (LAR 2) to find if a node is member of request zone or not.

1. LAR Box Protocol: It takes into account the location of $S$ and expected Zone for $D$, to check if a neighbor is within the request zone. To calculate the Expected Zone following information is used:

a. The most recent location on D i.e. (Xd, Yd).

b. The time at which the above location is obtained i.e. to.

c. The average velocity of $D$ i.e. Vavg.

d. The current time 11 .

With the help of these information, an expected circle with radius $\mathrm{R}=$ Vavg * (t1-t0), centered on (XD, YD) is obtained. The Request Zone is a rectangle area with Source $S$ in one comer (Xs, Ys), and the Expected Zone containing $D$ in the other corner. If a neighbor finds that, it is within the Request Zone, it forwards the route request packet further in the network. A node which is not a neighbor, knows that it is within the Request Zone by using the location of the neighbor (that sent the route request packet) and the Expected Zone for $D$.

2. LAR Step Protocol: It takes into account the distance. Suppose that the distance between current node $S$ and $D$ is $x$ and distance between neighbor I (which sent the route request packet) and $D$ is $y$. Now if $x<y$, then current node falls within the request zone. Hence it can forward the route request packet further.

\subsection{OLSR (Optimized Link State Routing) [3]}

Optimized Link state Routing protocol is a proactive routing protocol; hence the routes are always immediately available when needed. OLSR is an optimization version of a pure link state protocol. The change in topology causes flooding of information to all nodes. In order to reduce this overhead, Multi point relays (MPR) are used. MPR is used to:

1. Reduce the flooding by minimizing the same broadcast in a particular region.

2. Provide the shortest path.

For efficient routing four types of control messages are used:

a) Hello message is sent periodically to all neighbors node having node's identifier, list of node's neighbors, its MPRs and its neighbors. This is done to find updates about the link status and host's neighbor.

b) Topology Control (TC) massage is periodically sent by a node having a set of bidirectional links between the node and a subset of node's neighbors. Its purpose is to broadcast 
information about one's own neighbor. TC message is broadcasted periodically, with only MPR hosts forwarding it.

c) Multiple Interface Declaration (MID) message, is used to inform other nodes that the announcing host can have multiple OLSR interface. It is broadcasted only by MPR.

d) Host and Network Association (HNA) message, is used to give information regarding external routing. The HNA message contains vital information regarding the network and the net mask addresses. This information is used to announce that a particular node can act as a gateway.

\subsection{ZRP (Zone Routing Protocol) [4]}

It is a hybrid and hierarchical protocol, which means that it takes advantage of both reactive and proactive routing. It works on the principle of separation of nodes and local neighborhood from global topology of entire network. The above mentioned local neighborhoods are called zones. Important assumptions are:

1. Each node may be within a single zone or multiple overlapping zones.

2. Each zone may be of diverse size.

3. The routing zone has a radius $\rho$ (in hops).

The nodes of a zone are divided into 2 parts:

a) Peripheral nodes: Nodes which are equidistant to central node, with same radius $\rho$.

b) Interior nodes: Nodes which are non-equidistant to central node, with radius less than $\rho$.

In order to control the number of nodes in the routing zone, transmission power of the nodes is adjusted i.e. by lowering the power; number of nodes within direct reach is reduced.

ZRP utilizes two components:

1. At local level, IntrA-zone Routing Protocol (IARP) for Proactive routing component.

2. At global level, IntEr-zone Routing Protocol (IERP) for reactive routing component.

In ZRP, border casting is used instead of broadcasting. In order to direct queries to the border of the zone, the topology information provided by IARP is used. The Border cast Resolution Protocol (BRP) provides the packet delivery service in case of a border cast.

\section{Mobility Models}

\subsection{RWP (Random Way Point mobility model)}

It was proposed by Johnson and Maltz [5]. Due to its simplicity and availability at a wider scale, it took no time to become benchmark model. At the start of the simulation, each mobile node randomly selects one location in the simulation area as the destination and starts moving towards that destination with constant velocity which is chosen to be uniform and random between 0 to Vmax, where Vmax is the maximum velocity for a node. The velocity and direction of a node are independent of other nodes. When the destination is reached, the node rests for some time, called pause time Tpause. After this time elapse, again some other random destination is chosen and the process continues till the simulation ends. If Tpause $=0$, then there is no rest time and the mobility becomes continuous. The pause time Tpause, is chosen between [Tpausemin, Tpausemax].

\subsection{GM (Gauss Markov model)}

It was introduced by Liang and Haas [6]. Here the velocity of a node is assumed to be correlated over time and is modeled as a Gauss-Markov stochastic process. Using a stochastic formula, the path is 
Deepak Kumar and Suresh C. Gupta; Routing Protocols under different Mobility Models, Node Density and Speed, Transactions on Networks and Communications, Volume 3 No 1, Feb (2015); pp: 93-110

determined. When the node travels beyond the boundaries of simulation area, the direction of movement is forced to flip 180 degrees. In this manner, the boundary of simulation field is never touched. The velocity $\mathrm{Vt}$ at time $\mathrm{t}$ is dependent on velocity $\mathrm{Vt}-1$ at time $\mathrm{t}-1$. Hence temporal dependent property is exhibited. This degree of dependency is determined by parameter $\alpha$, which reflects the randomness of Gauss-Markov process i.e. if $\alpha=0$ a total randomness is observed and if $\alpha=1$ a total linear motion is observed. Hence we choose $\alpha$ between 0 to 1 according to the desired randomness. Initially, this model was used for the simulation of Personal Communication Service. But later on its use was extended to MANETs as well.

\subsection{RPGM (Reference point group mobility model)}

It was proposed by Hong et al. [7] in 1999. It was based on the thinking that nodes in MANET always tend to co-ordinate their movement. It takes care of the random movement of group and individual random movement of each node inside the group. Each group has a center, considered as group leader. The rest constitutes the group member.

Vtgroup represents the motion vector of group leader at time $t$. It defines the motion of group leader as well as the general motion trend of whole group. Because of this, the movement of group members is significantly affected. Mobility for each node is assigned with a reference point, which follows the group movement. Based on this reference point, each mobile node can be placed randomly in the neighborhood.

\subsection{MG (Manhattan Grid model)}

It was proposed by Bai et al. [8] in 2003. It models the mobility of nodes on streets defined by maps. The map consists of horizontal and vertical streets. Each street has two lanes for bidirectional moment. The mobile node is free to move along the horizontal and vertical lines in the grid. At the intersection of horizontal and Vertical Street, the mobile node can turn left or right or can go street. The choice is probabilistic i.e. turning left and right has a probability of 0.25 while probability for going straight is 0.5 . Velocity at any particular time $t$ is dependent on velocity at previous time $t-1$ and also on the velocity of node ahead on the same lane of the street.

\section{State of the Art}

Looking at the available literature, it is found that there are many studies done on MANET earlier. Some of them are very detailed while others are more technical. In late 90's researchers started working on MANETs, as these networks were thought of getting more useful in the future.

A very initial study was done by Das et al. [9] in 1998. They routing protocols were evaluated at packet level. The simulator used for this purpose was MaRS (Maryland Packet Simulator). They compared AODV, DSR, TORA, DSDV, EXBF and SPF. The observation was that although the routing load was lowered in new protocols, the link state and distance vector protocols gave better performance in terms of packet delivery and end to end delay. The work was extended for more cases [10] of node speed and density in 2000 with same set of protocols.

The same year authors [11] compared AODV, DSDV, DSR and TORA routing protocols on NS2 (Network Simulator 2). Random Way Point mobility model (RWP) was used for movement of nodes. For the above mentioned algorithms, the authors evaluated packet delivery ratio, routing overhead and path optimality. The node density was fixed at 50 .

An important work was done by X Hong et al. [12] in 1999. They presented a survey of mobility models in cellular and multi-hop networks. They showed that group motion occurs frequently in ad 
hoc environments, and based on this designed a group mobility model called RPGM (Reference Point Group Mobility model). They also showed that by changing the value of parameters in RPGM, many other mobility models can be modelled. They applied this mobility model to study the behavior on clustering and routing. The simulator used was parallel simulation language Maisie. The node density was fixed at 100 and protocols compared were DSDV, AODV and HSR. For the first time they showed that performance of routing protocol depends on the choice of mobility model.

In 2000, Lee et al. [13] compared the performance of multicast protocols in Ad hoc environment. The protocols comprised of AMROUTE, FLOODING, ODMRP, CAMP and AMRIS. The metrics obtained were packet delivery ratio, number of data packets transmitted per data packet delivered, number of control bytes transmitted per data bytes delivered and number of control and data packets transmitted per data packet delivered. The number of multicast nodes was 20 with speed from 0 $\mathrm{kmph}$ to $72 \mathrm{kmph}$. The simulator chosen was GloMoSim. Authors concluded that mesh based protocols outperforms tree based protocols.

Authors in [14] proposed two multi path techniques for DSR protocol. It utilizes disjoint paths. For simulation MARS simulator was used and node density was fixed at 60 . The mobility model was designed based on some pre-defined distribution. Performance metrics for simulation included fractions of packets dropped, end to end delay, number of route discoveries and routing load. The authors concluded that multipath routing is better than single path routing and if all the intermediate nodes are provided shortest paths, then the performance is slightly better than providing only source with alternate paths.

In 2002, a comparative study of CBR and TCP performance on OLSR and AODV protocols was done by T. Clausen et al. [15]. The variation was done for traffic, density and mobility. The common used traffic type for MANET is CBR, but the internet uses TCP. For a heterogeneous environment consisting of both, what will be the effect of TCP and CBR? Which will be preferred? The number of nodes was fixed at 50, and random waypoint mobility model was used. The simulator used was NS2. The metrics studied were control traffic overhead, delivery ratio, path length, delay, total transfer time and normalized routing load. The conclusion from the paper was, the protocols may perform comparatively when exposed to $C B R$, but when the same scenario is exposed to $T C P$, it significantly affects performance.

Barrett et al. [16] conducted a comparative analysis of IEEE 802.11, CSMA and MACA media access protocols. They considered only static ad hoc networks. The GloMoSim simulator was used to obtain number of received packets, average latency of each packet, long term fairness and throughput. They concluded that typically, all protocols degrade significantly at higher packet injection rate. Also, it happens rather sharply.

To analyze the impact of mobility on performance of routing protocols for ad hoc networks, a framework named IMPORTANT was proposed by F. Bai et al. [8]. The mobility models used were RWP, RPGM, Freeway mobility and Manhattan mobility model. The density of nodes was fixed at 40. NS 2 was used for simulation. The routing protocols considered were DSR, AODV and DSDV. The authors showed that performance of protocol shows drastic variations across mobility models. So the performance rankings of protocols will change with a change in mobility model.

An energy based performance comparison of AODV, DSR, TORA and DSDV was done by $B$. Chen et al. [17]. The mobility models employed were Random Waypoint, RPGM and Manhattan grid model. The simulator used was NS 2 and node density was fixed at 50. The authors concluded that reactive protocols are more sensitive to speed than proactive protocols. It is more challenging to route 
Deepak Kumar and Suresh C. Gupta; Routing Protocols under different Mobility Models, Node Density and Speed, Transactions on Networks and Communications, Volume 3 No 1, Feb (2015); pp: 93-110

packets over Manhattan grid model over the others. For group movement reactive protocols are better than proactive protocols.

T. Kunz [18] provided an in depth study of one to one and many to many communication in MANET. The protocols studied were unicast routing protocol (DSR and AODV), Multicast routing protocol (ADMR, ODMRP and Extension of AODV) and Broadcast protocols (FLOOD and BCAST). Simulations were conducted on NS2 and number of nodes was fixed at 50 . The performance metrics included packet delivery ratio and latency. The authors concluded that broadcast protocols, in particular BCAST perform well and that too without a high overhead.

A multilayer analysis of the influence of mobility models on AODV protocol was done by Gomez et al. [19]. The traffic flow was considered to be TCP. The performance analysis was done at three layers viz. physical layer, network layer and transport layer. The mobility models considered were RWP, Gauss Markov model, Manhattan Grid and RPGM model. The simulator used was NS2 and density of nodes was fixed at 20 . The authors concluded that higher speeds does not necessarily means lower throughput.

To implement the ant mobility model which is based on the actual movement of a group of ants, simulations were conducted by Liao et al. [20].The effect of this mobility model on DSDV, DSR and AODV is examined. For the worthiness of the model ant mobility is compared to random waypoint model for same set of protocols. The simulator used is NS2. The number of nodes for the simulation is 50. The metrics evaluated were packet throughput ratio, average end to end delay and normalized routing load. The authors concluded that trace models like ant mobility are more accurate than synthetic models like random waypoint mobility. But this accuracy comes at a cost of difficult and time consuming process.

Atsan et al. [21] classified and compared the performance of mobility model for MANET protocols. The protocol studied was AODV and simulator chosen was SWANS. Four mobility models were considered viz. random direction, boundless simulation area model, random walk and random waypoint model. The metrics considered were average message activity, average route request completion rate and average RREQ message sent per route added. The density of node was fixed at 50. Authors concluded that although RAP does not give the best performance for all the used performance metrics, it is most consistent for varying simulation levels.

A realistic simulation based study of MANET protocols was made by Marinoni et al. [22]. They proposed a new and realistic Urban Mobility Model (UMM), which models realistic user motion and signal propagation in a city like scenario. The mobility models namely RWP, UMMoff (UMM with radio constraints activated) and UMMon (UMM with radio constraints deactivated) were applied on DSR protocol. The number of sender/receiver was 20 pairs for all experiments. NS2 was used for simulation. The metrics calculated were packet delivery ratio, end to end delay, path length and routing overhead. The authors concluded that trivial RWP is too simplistic and too narrow in its scope. Hence a realistic model like UMM can be a better choice.

Pirzada et al. [23] compared performance of multi-path AODV and DSR protocols in hybrid mesh networks.NS2 was the preferred simulator. The number of mesh clients was fixed at 50 and number of mesh routers was fixed at 16. Random waypoint model was considered for mobility. Packet loss, aggregate good put, packet delivery percentage, routing packet overhead, average latency and path optimality were the metrics calculated. The authors concluded that mesh networks with inclusion of mesh router gives better performance. 
G. Jayakumar et al. [24, 25] compared performance of DSR and AODV for random waypoint and Manhattan grid model. The node density was fixed at 20 nodes. The performance metrics included packet delivery fraction, average end to end delay, normalized routing load and normalized mac load. The simulator used was NS2. The authors observed a very clear trend between mobility metric, connectivity and performance.

In 2009 Karthikeyan et al. [26] studied the performance of broadcasting methods in MANET. The techniques employed for broadcasting was simple flooding and probability based flooding. The simulations were performed on NS2. The number of mobile nodes was fixed at 24 . The performance metric included normalized routing load for DSDV protocol. The authors concluded that probabilistic broadcast performs better than simple flooding.

A comparative performance analysis of DSDV, AODV and DSR routing protocols was done by Tuteja et al. [27]. For simulation, NS2 was used. The metrics included packet delivery ratio, throughput, end to end delay and routing overhead. 25 nodes were considered for simulation. Random waypoint model was used to define movements of node. The authors concluded that with the increase in mobility of nodes performance degrades irrespective of the choice of three discussed protocols.

Unicast and broadcast routing protocols of MANET were evaluated by Debnath et al. [28]. Both one to one and many to many communications were addressed in detail. DSR and BCAST protocol were simulated on NS2. The number of nodes was fixed at 50. Mobility model used was random waypoint mobility model. The performance metrics included packet delivery ratio, packet latency, normalized routing load, normalized mac load and throughput. The authors concluded that BCAST protocol works well in most scenarios and is robust even with high traffic environments.

Barakovic et al. [29] compared the performance of MANET routing protocols AODV, DSR and DSDV. Simulations were carried on NS2. Packet delivery ratio, average end to end delay and normalized routing load were the performance metrics. The numbers of source nodes varied from 10 to 30 . The mobility of nodes was defined by random waypoint model. The conclusion form the study was that all the protocols reacted in similar ways for low mobility and low load conditions, while DSR outperformed AODV and DSDV with increasing mobility and load.

In 2011, Mohapatra et al. [30] studied the effect of change in network size, mobility and pause time on AODV, OLSR and DSDV. The number of nodes was fixed at 30. The choice of simulator was NS2 and that of mobility model was random waypoint model. Throughput, Routing overhead, delay and packet delivery ratio were calculated for varying number of nodes, varying pause time and varying network area. The authors concluded that for highly mobile random network OLSR is preferred.

Performance comparison of relatively newer set of protocols viz. LANMAR, LAR1, DYMO and ZRP was done by Singh et al. [31]. Qualnet simulator was chosen for the experiments. 50 nodes were considered for the scenario. Random waypoint model was used to define the mobility pattern of nodes. The performance metrics were average end to end delay, packet delivery ratio, throughput and average jitter. The authors concluded that LANMAR is the best scheme in terms of end to end delay and jitter while LAR1 is best in terms of packet delivery ratio and throughput.

A comparative study was done by Saada et al. [32] to evaluate the performance of protocols. GloMoSim simulator was used for experiments. To compare the performance DSDV, AODV, ARPM and SHARP protocols were considered. The number of nodes was different for different scenarios. For static scenario, it was fixed at 70 while for dynamic scenario it varied from 10 to 140. Random waypoint model was the mobility model and the metrics included overhead, route discovery delay 
Deepak Kumar and Suresh C. Gupta; Routing Protocols under different Mobility Models, Node Density and Speed, Transactions on Networks and Communications, Volume 3 No 1, Feb (2015); pp: 93-110

and throughput. The conclusion derived from the work was that DSDV is better for small networks and AODV is better for large networks.

From the above given analysis, we conclude that although a lot of comparative studies have been carried out on MANET routing protocols based on one or more mobility models, most of them have relied on Random waypoint model, which due to its probabilistic nature is unrealistic. Most of the work has been done considering either variation in node density or node speed along with mobility models. We have considered variation in speed and density of nodes together as a parameter to study the effects on a wider perspective. Also for the choice of mobility models we have considered RWP, MG, GM and RPGM. We have taken a candidate protocol from each group of protocols viz. reactive, proactive, location based and geographic.

\section{Simulation Setup}

To study the performance of routing protocols we evaluated throughput, end to end delay and ratio of packet loss. The metrics are described as follows.

- Throughput: It is the ratio of number of packets received at destination to the number of packets originated at source. The source follows CBR (Constant bit rate) traffic. It depicts the loss rate.

Throughput $=$ Data packets received / Data packets sent

- End to end delay: It is the average amount of time that is taken by a packet to reach final destination from source. It includes the route discovery wait time, which a node may experience in case a route is not available.

Average delay $=\Sigma(\operatorname{tr}-\mathrm{ts}) / \mathrm{Pr}$, where ts is the packet send time and $\operatorname{tr}$ is the packet receive time.

- Packet loss: It is the fraction of packet lost on their route to destination. The loss is usually due to congestion on the network and buffer overflows.

Packet loss $=$ Number of lost packets $/$ number of received packets

To generate mobility patterns for MG, RWP, GM and RPGM Bonn Motion tool is used. We have studied the impact of speed and node density on performance of the network. To compare the protocols, same set of scenarios is utilized for each one. The simulator used is GloMoSim [33]. The simulation parameters are given below.

\begin{tabular}{|c|c|}
\hline Parameter Name & Value \\
\hline Speed of node & 0 to $20 \mathrm{~m} / \mathrm{s}$ \\
\hline Density of node & 5 to 200 \\
\hline Number of CBR sources & 10 \\
\hline Speed of CBR link & 10 packets per second \\
\hline Packet Size & 512 bytes \\
\hline Wireless Radio & 802.11 \\
\hline Transmission Range & $50 \mathrm{~m}$ \\
\hline Transmission rate & $1 \mathrm{Mbps}$ \\
\hline Area of simulation & $1500 \mathrm{~m} \times 1500 \mathrm{~m}$ \\
\hline Simulation time & 300 seconds \\
\hline
\end{tabular}


The parameters chosen for mobility models are as follows:

\begin{tabular}{|c|c|c|}
\hline Model & Parameter & Value(s) \\
\hline RWP (Random Way Point) & Pause time & $0 \mathrm{sec}$ \\
\hline & Min. speed & $0 \mathrm{~m} / \mathrm{s}$ \\
\hline & Max. Speed & $20 \mathrm{~m} / \mathrm{s}$ \\
\hline MG (Manhattan Grid) & No. of blocks along y-axis & 2 \\
\hline & No. of blocks along x-axis & 10 \\
\hline & Min. Speed & $0 \mathrm{~m} / \mathrm{s}$ \\
\hline & Max. Speed & $20 \mathrm{~m} / \mathrm{s}$ \\
\hline & Probability of going straight & 0.5 \\
\hline & Probability of going right & 0.25 \\
\hline & Probability of going left & 0.25 \\
\hline RPGM (Reference Point & Average no. of nodes per group & 5 \\
\hline Group Mobility) & & \\
\hline & Max. distance to center of group & $5 \mathrm{~m}$ \\
\hline & Min. Speed & $0 \mathrm{~m} / \mathrm{s}$ \\
\hline & Max. Speed & $20 \mathrm{~m} / \mathrm{s}$ \\
\hline GM (Gauss Markov) & Min. Speed & $0 \mathrm{~m} / \mathrm{s}$ \\
\hline & Max. Speed & $20 \mathrm{~m} / \mathrm{s}$ \\
\hline
\end{tabular}

6 Results and Discussion

\subsection{Throughput}

LAR, OLSR, DSR and ZRP were tested under RWP, MG, RPGM and GM models. We varied the speed of the nodes from 0 to $20 \mathrm{~m} / \mathrm{s}$ at interval of 5 . The node density was varied from 50 to 200 in the intervals of 50. From the results, it is clear that LAR and OLSR outperform others in terms of throughput. The results are given in Fig. 1 to 4 . Throughput metrics is almost equal to $100 \%$ for OLSR and LAR. But, in the case of random waypoint model although OLSR still outperforms others but the throughput is reduced largely. This is due to the fact that in random waypoint model link breakage is more often for higher speeds and hence the throughput decreases for almost all protocols. At higher speeds i.e. 10 to $20 \mathrm{~m} / \mathrm{s}$ LAR and OLSR are the preferred choice for better throughput. But, at lower speeds the case changes, LAR behaves better than others for RWP and RPGM. Also, the applications which utilize RWP should use LAR for lower speeds. While the applications, which uses other mobility models can either opt for OLSR or LAR for all cases of mobility and speed.

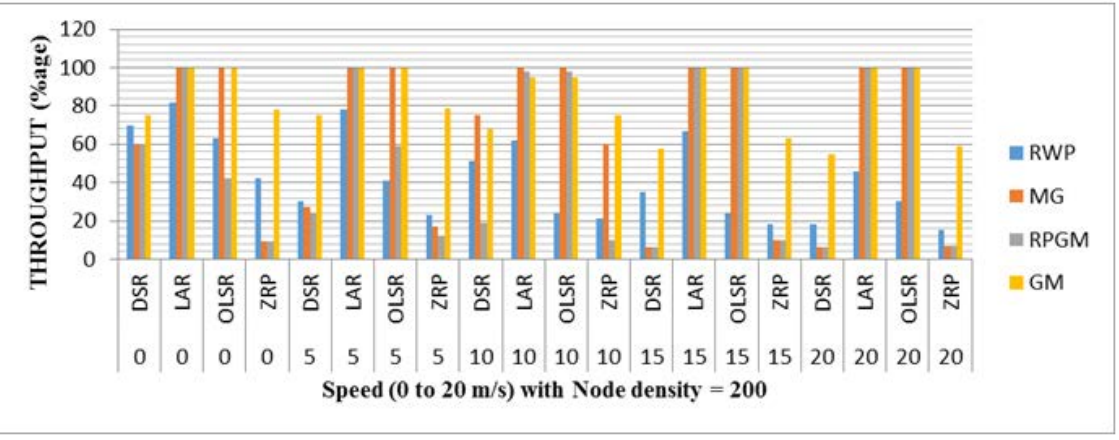

Figure 1: Throughput at Node Density 200, with speed varying from 0 to $20 \mathrm{~m} / \mathrm{s}$ 


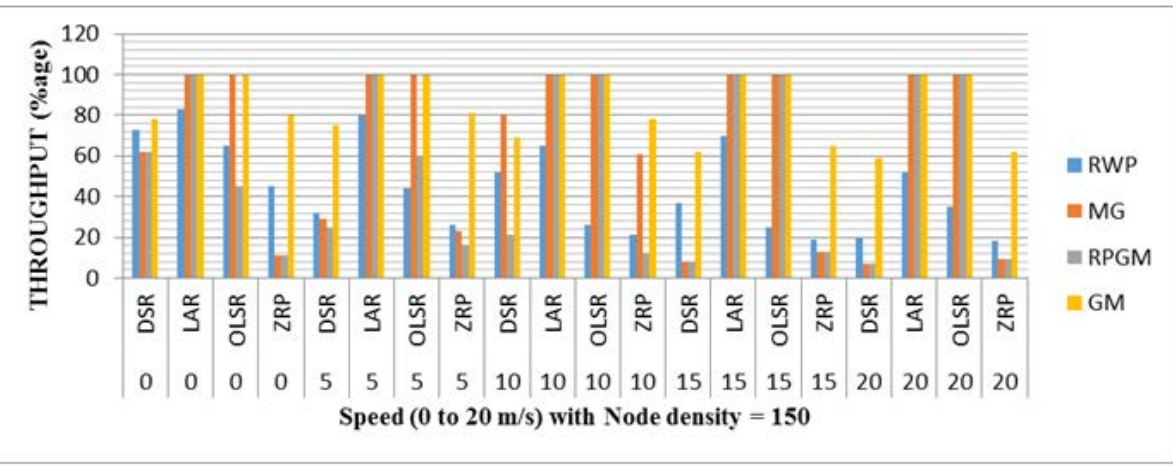

Figure 2: Throughput at Node Density 150, with speed varying from 0 to $20 \mathrm{~m} / \mathrm{s}$

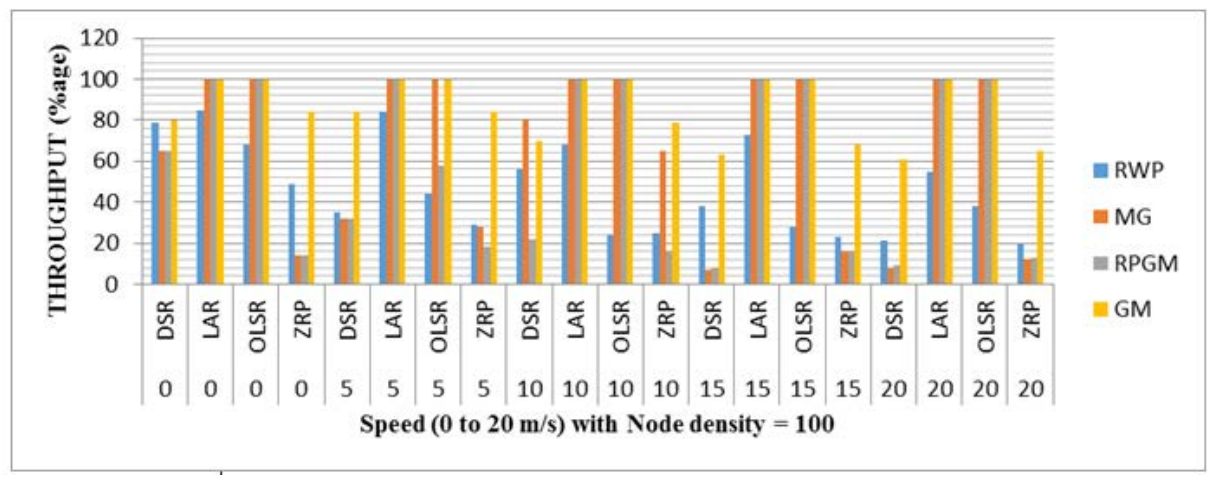

Figure 3: Throughput at Node Density 100, with speed varying from 0 to $20 \mathrm{~m} / \mathrm{s}$

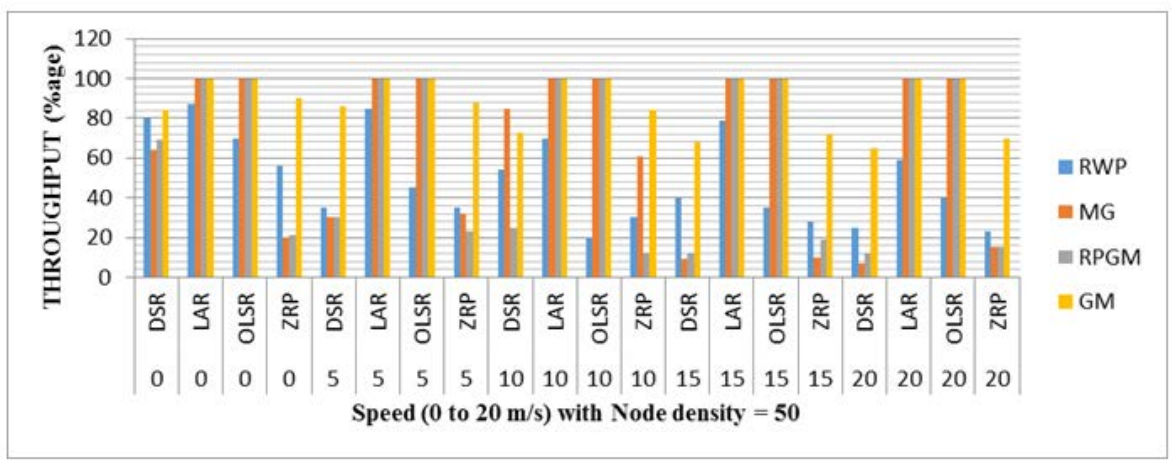

Figure 4: Throughput at Node Density 50, with speed varying from 0 to $20 \mathrm{~m} / \mathrm{s}$

\subsection{End to End Delay}

The results for end to end delay are shown in Fig. 5 to 8. The values for delay for some case is very small, and to make that portion visible additional sub graph is given, which highlights the smaller values. These sub graphs are numbered from $5 \mathrm{a}$ to $8 \mathrm{a}$. The speed of the nodes is varied from 0 to 20 $\mathrm{m} / \mathrm{s}$ in steps of 5 and node density is varied from 50 to 200 in steps of 50 . LAR exhibits lowest end to end delay for almost all the cases of speed and node density compared to other protocols. With increase in speed and number of nodes the delay also increases. It happens because at higher speeds, connectivity decreases and hence accounts for higher delays. The applications which require less end to end delay should use LAR, as is clear from the above discussion. 


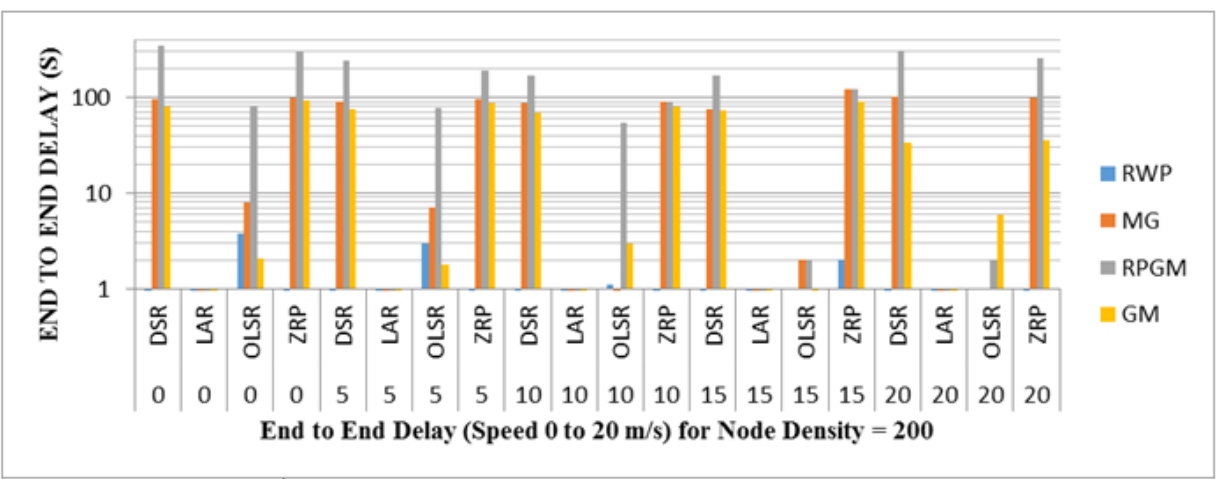

Figure 5: End to End Delay at Node Density 200, with speed varying from 0 to $20 \mathrm{~m} / \mathrm{s}$

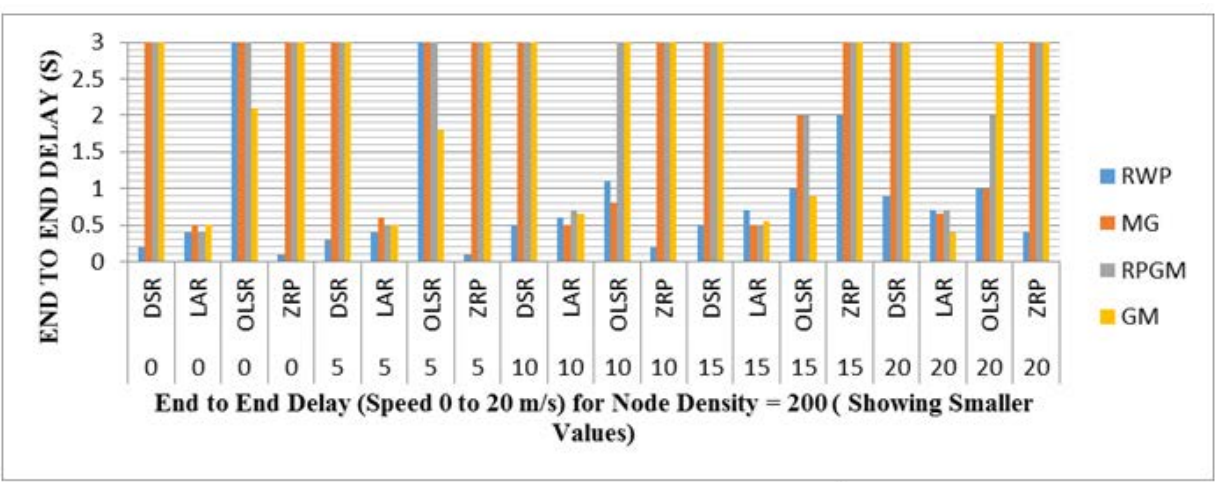

Figure 5a: End to End Delay at Node Density 200, speed varying from 0 to $20 \mathrm{~m} / \mathrm{s}$ (Only smaller values are shown)

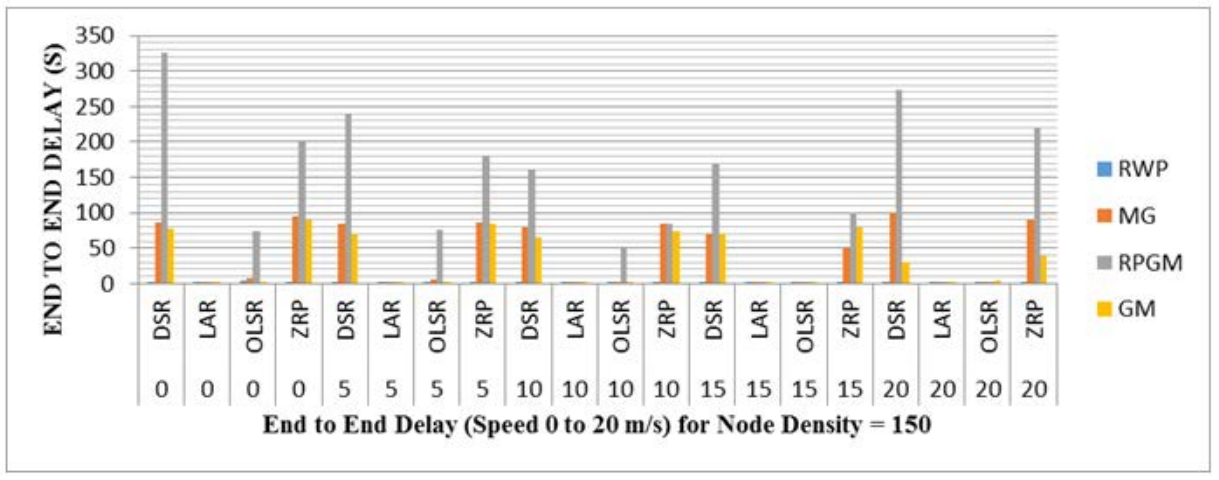

Figure 6: End to End Delay at Node Density 150, with speed varying from 0 to $20 \mathrm{~m} / \mathrm{s}$

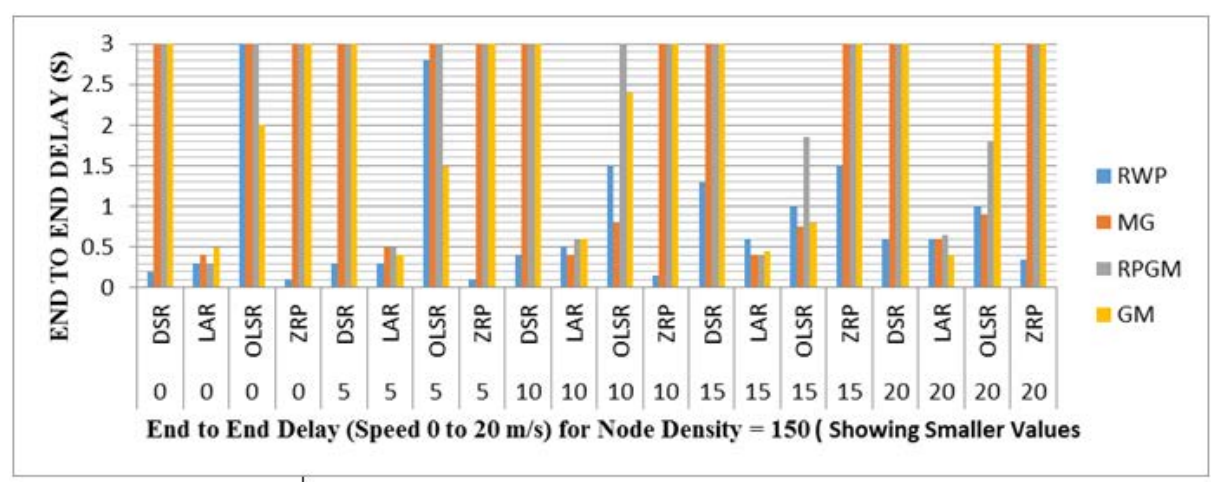

Figure 6a: End to End Delay at Node Density 150, speed varying from 0 to $20 \mathrm{~m} / \mathrm{s}$ (Only smaller values are shown) 


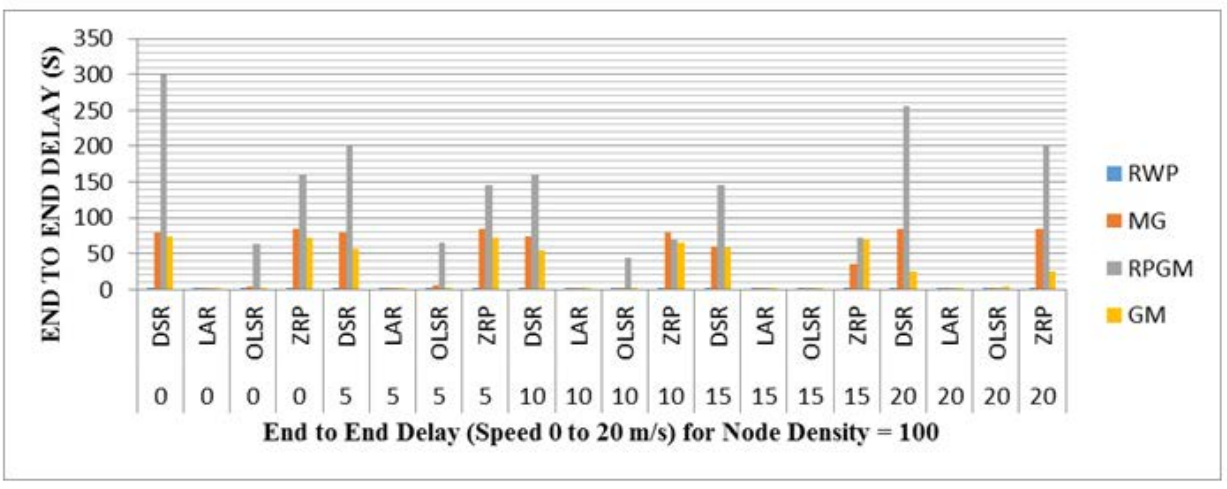

Figure 7: End to End Delay at Node Density 100, with speed varying from 0 to $20 \mathrm{~m} / \mathrm{s}$

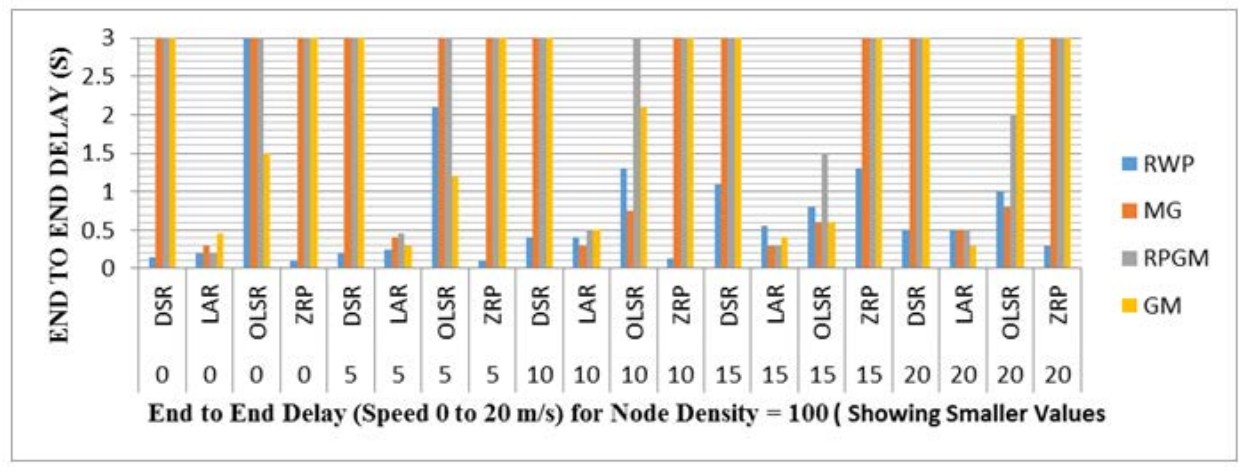

Figure 7a: End to End Delay at Node Density 100, speed varying from 0 to $20 \mathrm{~m} / \mathrm{s}$ (Only smaller values are shown)

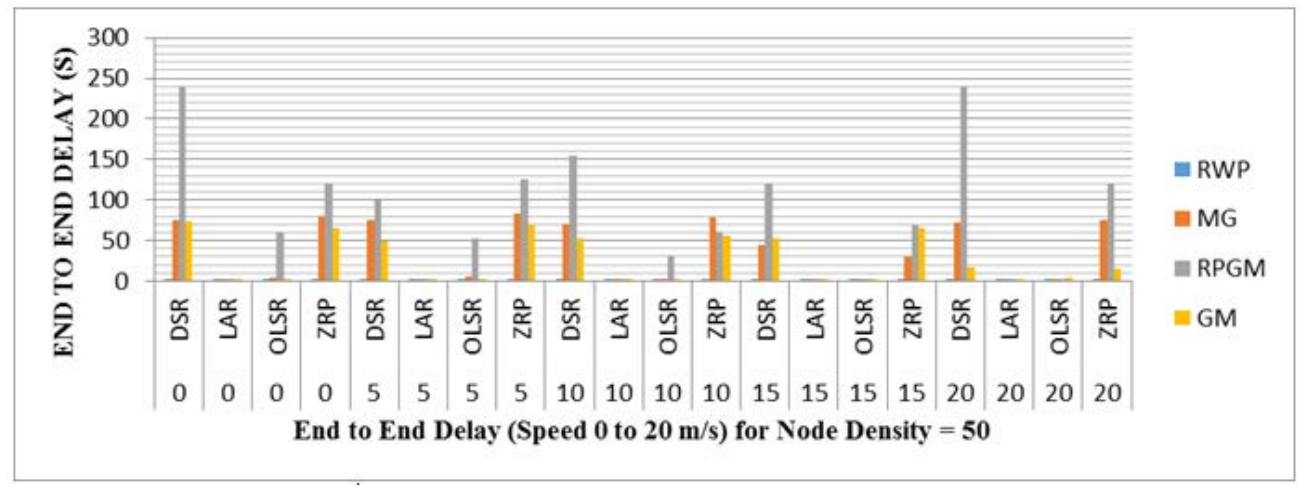

Figure 8: End to End Delay at Node Density 50, with speed varying from 0 to $20 \mathrm{~m} / \mathrm{s}$

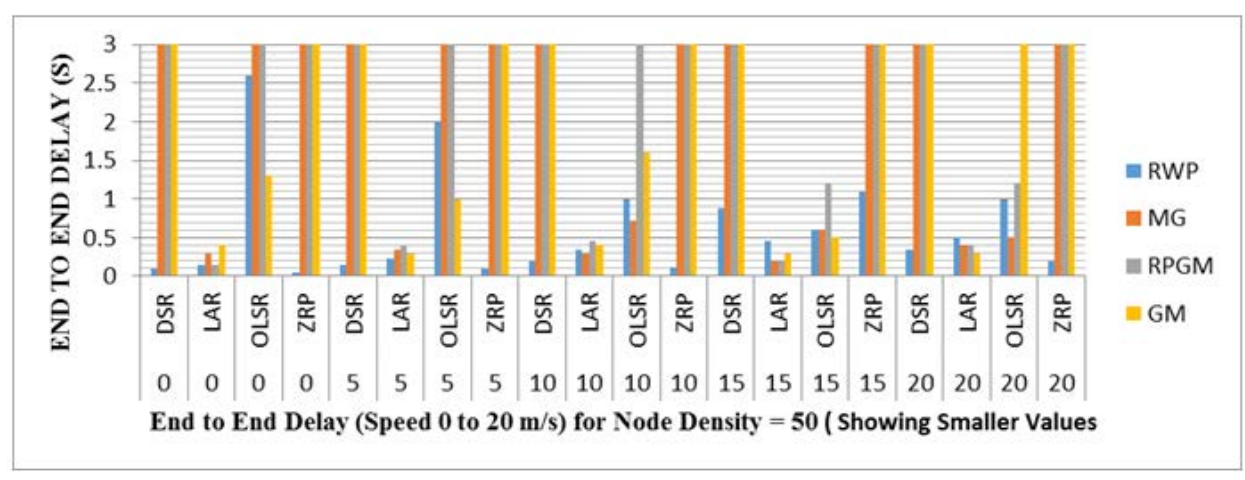

Figure 8a: End to End Delay at Node Density 50, speed varying from 0 to $20 \mathrm{~m} / \mathrm{s}$ (Only smaller values are shown 


\subsection{Packet Loss}

The results for packet loss are shown in fig 9 to 12. The speed of the nodes is varied from 0 to $20 \mathrm{~m} / \mathrm{s}$ in steps of 5 and node density is varied from 50 to 200 in steps of 50. At all node density, Gauss Markov model gives the lowest packet loss for all protocols, specially LAR and OLSR. This can be due to the fact that the value of speed and direction at the nth location is dependent on the previous value and a random variable. It means that probability of a node to remain in its old entirety is more, hence incurring low packet loss. LAR has the least packet loss with respect to other protocols for almost all cases of node speed and density except random way point model. With increase in speed the probability of packet loss also increases. It happens because at higher speeds, connectivity decreases and hence accounts for packet loss delays. The applications which require less packet loss should use LAR, as is evident from the results.shown)

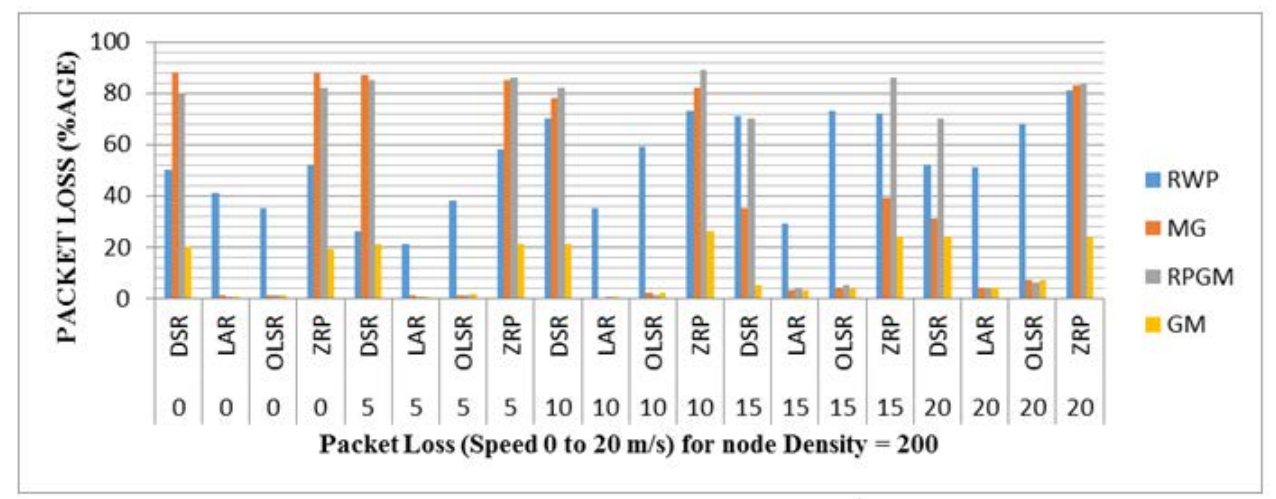

Figure 9: Packet Loss at Node Density 200, with speed varying from 0 to $20 \mathrm{~m} / \mathrm{s}$

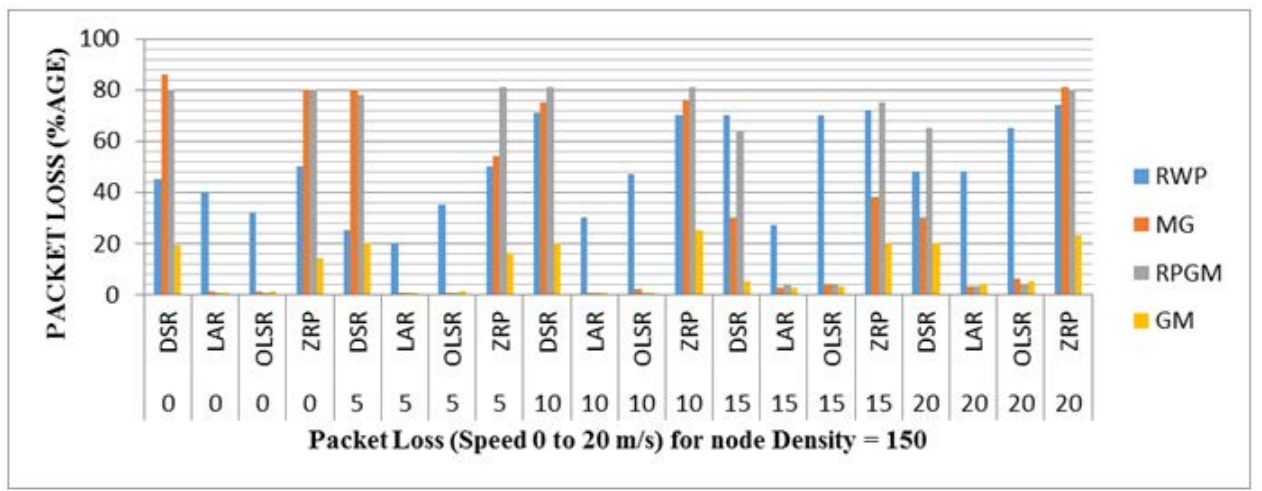

Figure 10: Packet Loss at Node Density 150, with speed varying from 0 to $20 \mathrm{~m} / \mathrm{s}$

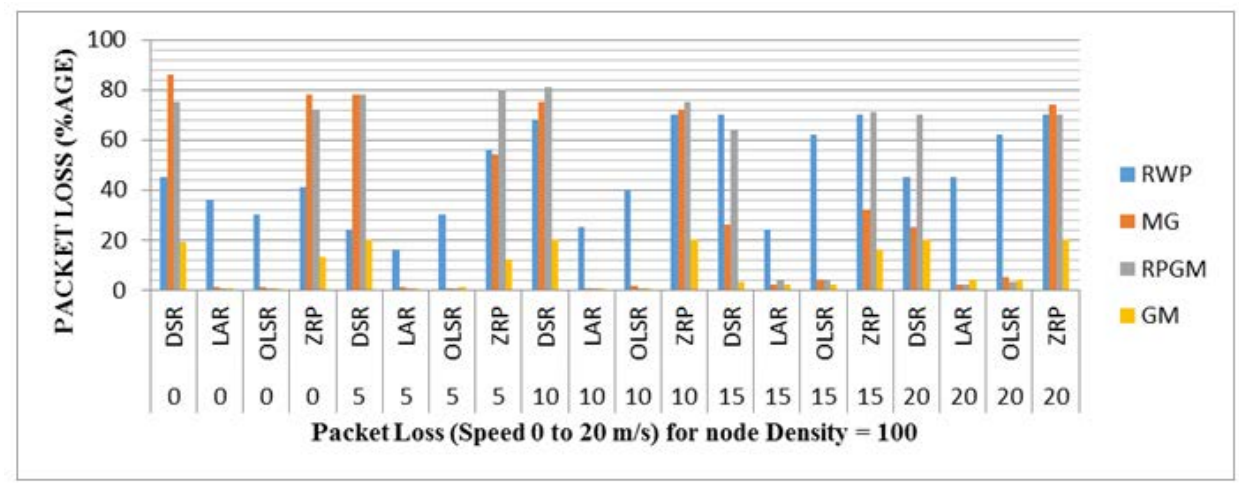

Figure 11: Packet Loss at Node Density 100, with speed varying from 0 to $20 \mathrm{~m} / \mathrm{s}$ 


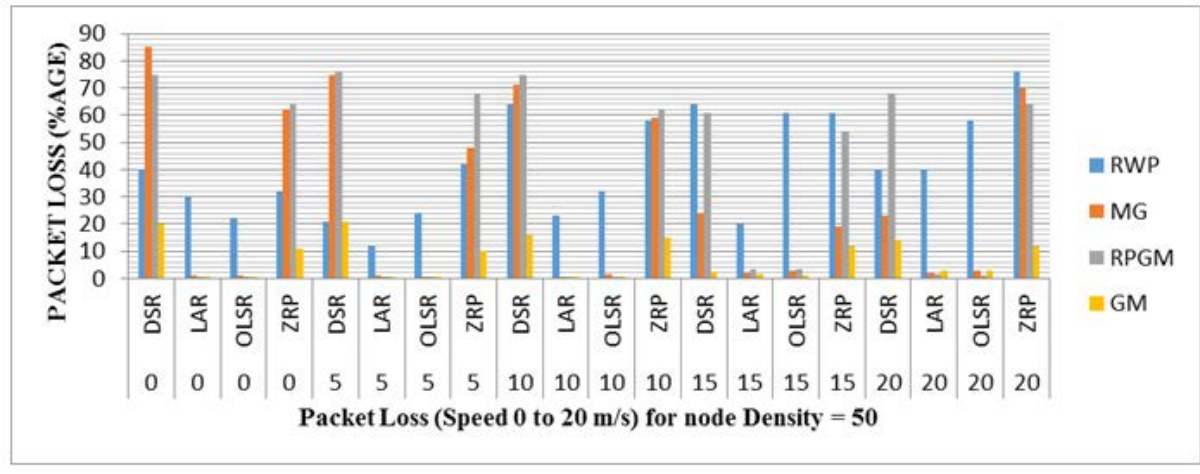

Figure 12: Packet Loss at Node Density 50, with speed varying from 0 to $20 \mathrm{~m} / \mathrm{s}$

\section{Conclusions}

In this work we presented a simulation based performance analysis of MANET routing protocols. From the reactive, proactive, geographic and location based protocols, one candidate protocol was chosen for analysis. The mobility models used were random way point, gauss Markov, Manhattan Grid and reference point group mobility model. The variation in node speed was done from 0 to 20 $\mathrm{m} / \mathrm{s}$ and node density from 50 to 200 . The analysis of the throughput suggests that LAR and OLSR with MG model gives $100 \%$, due to the fact that a restriction in mobility area in a grid betters the throughput. LAR and OLSR give best performance in RPGM, due to the presence of a group leader, who is responsible for the mobility of the group and even distribution of group members. Due to localization property LAR performs better under RWP. This is due to the fact that in RWP model the nodes are distributed such that they are able to move freely and independently of others.

\section{REFERENCES}

[1] D. Johnson, Y. Hu, D. Maltz, "The Dynamic Source Routing Protocol (DSR) for mobile Ad Hoc Networks for IPV4", rfc4728.

[2] Young-Bae Ko, Nitin H. Vaidya, "Location-Aided Routing (LAR) in mobile ad hoc networks", Wireless Networks, Volume 6, Issue 4, pp 307-321.

[3] T. Clausen, P. Jacquet, “Optimized Link State Protocol (OLSR)”, rfc3626.

[4] Zygmunt J. Haas, Marc R. Pearlman, Prince Samar, "The Zone Routing Protocol for Adhoc networks", draft-ietf-manet-zone-zrp-04.txt, july2002

[5] J. Broch, D. A. Maltz, D. B. Johnson, Y.C. Hu, and J. Jetcheva, "A performance comparison of multi-hop wireless ad hoc network routing protocols", in Proceedings of the Fourth Annual ACM/IEEE International Conference on Mobile Computing and Networking(Mobicom98), ACM, October 1998.

[6] B. Liang, Z. J. Haas, "Predictive Distance-Based Mobility Management for PCS Networks", in Proceedings of IEEE Information Communications Conference (INFOCOM 1999), Apr. 1999.

[7] X. Hong, M. Gerla, G. Pei, and C.C. Chiang, "A group mobility model for ad hoc wireless networks", in Proceedings of ACM International Workshop on Modeling, Analysis, and Simulation of Wireless and Mobile Systems (MSWiM), August 1999. 
[8] F. Bai, N. Sadagopan, and A. Helmy, "Important: a framework to systematically analyze the impact of mobility on performance of routing protocols for ad hoc networks", in Proceedings of IEEE Information Communications Conference (INFOCOM 2003), San Francisco, Apr. 2003.

[9] 10. Samir R. Das, Robert Castaneda, Jiangtao Yan, and Rimli Sengupta, "Comparative Performance Evaluation of Routing protocols for Mobile, Ad hoc Networks", in Proceedings of Seventh international Conference on Computer Communication and Networks (IC3N), Lafayette, LA, October 1998, pp. 153-161.

[10] Samir R. Das, Robert Castaneda, and Jiangtao Yan, "Simulation-based performance evaluation of routing protocols for mobile ad hoc networks", Journal of Mobile Networks and Applications, Volume 5, pp. 179-189, 2000.

[11] Josh Broch, David A. Maltz, David B. Johnson, Yih-Chun Hu, and Jorjeta Jetcheva, "A Performance Comparison of Multi-Hop Wireless Ad Hoc Network Routing Protocols", in Proceedings of the Fourth Annual ACM/IEEE International Conference on Mobile Computing and Networking (MobiCom'98), October 25-30, 1998, Dallas, Texas, USA.

[12] Xiaoyan Hong, Mario Gerla, Guangyu Pei, Ching-Chuan Chiang, "A group mobility model for ad hoc wireless networks", in Proceeding of the 2nd ACM international workshop on Modeling, analysis and simulation of wireless and mobile systems, MSWiM '99, ACM New York, NY, USA, pp. 53-60.

[13] Sung-Ju Lee, W. Su, J. Hsu, M. Gerla, R. Bagrodia, "A performance comparison study of ad hoc wireless multicast protocols" in proceedings of Nineteenth Annual Joint Conference of the IEEE Computer and Communications Societies, INFOCOM 2000, pp. 565-574.

[14] Asis Nasipuri, Robert Castaneda, Samir R. Das, "Performance of Multipath Routing for OnDemand Protocols in Mobile Ad Hoc Networks", Journal of Mobile Networks and Applications, Volume 6, pp. 339-349, 2001,Kluwer Academic Publishers.

[15] Thomas Clausen, Philippe Jacquet, and Laurent Viennot, "Comparative study of CBR and TCP performance of MANET routing protocols", in Workshop on Broadband Access, INRIA Lab, France, Sep. 2002.

[16] C. L. Barrett, M. Drozda, and M.V. Marathe, "A comparative experimental study of media access protocols for wireless radio networks", Wireless Communications and Networking Conference (WCNC 2002), 17-21 Mar 2002, pp. $405-411$.

[17] Bor-rong Chen and C. Hwa Chang, "Mobility Impact on Energy Conservation of Ad Hoc Routing Protocols", SSGRR 2003, Italy, Jul. 28-Aug. 2, 2003.

[18] Thomas Kunz, "Reliable Multicasting in MANETs", Contract Report, DRDC-Ottawa, July 2003.

[19] C. Gomez, X. Marchador, V. Gonzalez, and J. Paradells, "Multilayer analysis of the influence of mobility models on TCP flows in AODV ad-hoc networks", in Proceedings of the 14th 
IEEE Workshop on Local and Metropolitan Area Networks (LANMAN), 2005, 18 Sept. 2005, pp 1-6.

[20] Hsien-Chou Liao, Yi-Wei Ting, Chia-Meng Chen, and Chou-Chen Yang, "A Performance Comparison of Ad Hoc Routing Protocols Based on Ant Mobility Model", Information Technology Journal, Vol. 4, No. 3, 2005, pp. 278-283.

[21] Emre Atsan, Oznur Ozkasap, "A Classification and Performance Comparison of Mobility Models for Ad Hoc Networks", Ad-Hoc, Mobile, and Wireless Networks, Lecture Notes in Computer Science Volume 4104, 2006, pp 444-457.

[22] Stefano Marinoni, Hannu H. Kari, "Ad hoc routing protocol's performance: a realistic simulation based study", Telecommunication Systems, December 2006, Volume 33, Issue 1-3, pp 269-289.

[23] Asad Amir Pirzada, Marius Portmannt, and Jadwiga Indulska, "Performance Comparison of Multi-Path AODV and DSR Protocols in Hybrid Mesh Networks", in Proceedings of 14th IEEE International Conference on Networks (ICON), Sept. 2006, pp. 1-6.

[24] Geetha Jayakumar, and G. Gopinath, "Performance Comparison of Two On-demand Routing Protocols for Ad-hoc Networks based on Random Way Point Mobility Model", American Journal of Applied Sciences, Vol. 5, Issue 6,2008, pp. 659-664.

[25] Geetha Jayakumar, and G. Gopinath, "Performance comparison of MANET protocols based on Manhattan Grid Mobility Model", Journal of Mobile Communication, Vol. 2, Issue 1, 2008, pp. 18-26.

[26] N.Karthikeyan, V. Palanisamy, and K. Duraiswamy, "Performance Comparison of Broadcasting methods in Mobile Ad Hoc Network", International Journal of Future Generation Communication and Networking, Vol. 2, No. 2, June 2009, pp. 47-58.

[27] Asma Tuteja, Rajneesh Gujral, and Sunil Thalia, "Comparative Performance Analysis of DSDV, AODV and DSR Routing Protocols in MANET using NS2", in Proceedings of International Conference on Advances in Computer Engineering (ACE), 20-21 June 2010, pp. $330-333$.

[28] Sumon Kumar Debnath, Foez Ahmed, and Nayeema Islam, "Performance Evaluation of Unicast and Broadcast Mobile Ad-hoc Networks Routing Protocols", International Journal of Computer Science and Information Security (IJCSIS), Vol. 7, No. 1, 2010, pp. 40-46.

[29] Sabina Barakovi, Jasmina Barakovi, "Comparative Performance Evaluation of Mobile Ad Hoc Routing Protocols", MIPRO 2010, May 24-28, 2010, Opatija, Croatia, 518-523.

[30] S. Mohapatra, P. Kanungo, "Comparative Performance Analysis of MANET Routing Protocols Using NS2 Simulator", Computational Intelligence and Information Technology, Communications in Computer and Information Science, Volume 250, 2011, pp 731-736. 
[31] Dinesh Singh, Ashish K. Maurya, Anil K. Sarje, "Comparative Performance Analysis of LANMAR, LAR1, DYMO and ZRP Routing Protocols in MANET using Random Waypoint Mobility Model", in proceedings of 3rd International conference on Electronics Computer Technology (ICECT), 8-10 April 2011, pp. 62-66.

[32] Imad I. Saada, Raed Zaghal, "MANET Routing Protocols: Comparative Study", Journal of AlQuds Open University for Research and Studies, Vol. 30, Part 1, June 2013, pp. 9-26.

[33] X. Zeng, R. Bagrodia, and M. Gerla, "GloMoSim A Library for Parallel Simulation of LargeScale Wireless Networks", in Proceedings of the 12th ACM Workshop on Parallel and Distributed Simulation, Canada, 1998, pp. 154-161. 